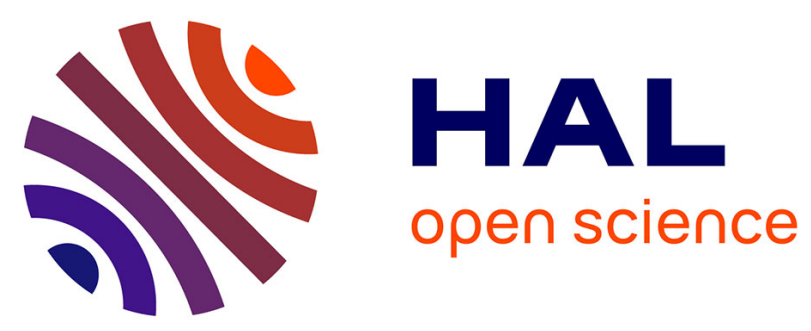

\title{
From Euclidean to Riemannian Means: Information Geometry for SSVEP Classification
}

Emmanuel Kalunga, Sylvain Chevallier, Quentin Barthélemy, Karim Djouani, Yskandar Hamam, Eric Monacelli

\section{- To cite this version:}

Emmanuel Kalunga, Sylvain Chevallier, Quentin Barthélemy, Karim Djouani, Yskandar Hamam, et al.. From Euclidean to Riemannian Means: Information Geometry for SSVEP Classification. Geometric Science of Information, Oct 2015, Palaiseau, France. pp.595-604, 10.1007/978-3-319-25040-3_64 . hal-01351753

\section{HAL Id: hal-01351753 \\ https://hal.science/hal-01351753}

Submitted on 4 Aug 2016

HAL is a multi-disciplinary open access archive for the deposit and dissemination of scientific research documents, whether they are published or not. The documents may come from teaching and research institutions in France or abroad, or from public or private research centers.
L'archive ouverte pluridisciplinaire HAL, est destinée au dépôt et à la diffusion de documents scientifiques de niveau recherche, publiés ou non, émanant des établissements d'enseignement et de recherche français ou étrangers, des laboratoires publics ou privés.

\section{(c)(1)}

Distributed under a Creative Commons Attribution| 4.0 International License 


\title{
From Euclidean to Riemannian Means: Information Geometry for SSVEP Classification
}

\author{
Emmanuel K. Kalunga ${ }^{1,2}$, Sylvain Chevallier ${ }^{2}$, Quentin Barthélemy ${ }^{3}$, \\ Karim Djouani ${ }^{1}$, Yskandar Hamam ${ }^{1}$, and Eric Monacelli ${ }^{2}$ \\ 1 Department of Electrical Engineering/F'SATI \\ Tshwane University of Technology, Pretoria 0001, South Africa \\ ${ }^{2}$ Laboratoire d'Ingénierie des Systèmes de Versailles, \\ Université de Versailles Saint-Quentin, 78140 Velizy, France \\ 3 Mensia Technologies, ICM, Hôpital de la Pitié-Salpêtrière, \\ 75013 Paris, France \\ emmanuelkalunga.k@gmail.com, sylvain.chevallier@uvsq.fr \\ qb@mensiatech.com, djouanik@tut.ac.za \\ yskandar@hamam.ws, eric.monacelli@uvsq.fr
}

\begin{abstract}
Brain Computer Interfaces (BCI) based on electroencephalography (EEG) rely on multichannel brain signal processing. Most of the state-of-the-art approaches deal with covariance matrices, and indeed Riemannian geometry has provided a substantial framework for developing new algorithms. Most notably, a straightforward algorithm such as Minimum Distance to Mean yields competitive results when applied with a Riemannian distance. This applicative contribution aims at assessing the impact of several distances on real EEG dataset, as the invariances embedded in those distances have an influence on the classification accuracy. Euclidean and Riemannian distances and means are compared both in term of quality of results and of computational load.
\end{abstract}

Keywords: Information geometry, Riemannian means, Brain-Computer Interfaces, Steady State Visually Evoked Potentials.

\section{Introduction}

Brain-Computer Interfaces (BCI) allow interaction with a computer or a machine without relying on the user's motor capabilities. In rehabilitation and assistive technologies, BCI offer promising solutions to compensate for physical disabilities. To record brain signals in BCI systems, the most common choice is to rely on electroencephalography (EEG) [15], as the recording systems are smaller and less expensive than other brain imaging technologies (such as MEG or fMRI). BCI systems rely on different brain signals, such as event-related desynchronization or evoked potentials. The former is observed in the premotor cortex when the subject imagines moving some part of his own body (also known as Motor Imagery paradigm) and the latter qualifies the brain response to a specific sensory stimulation, usually visual or auditory. This contribution focuses on 
Steady-State Visually Evoked Potentials (SSVEP), which are potentials emerging when a subject concentrates his attention on a stimulus blinking at a given frequency. Shortly after the user concentrates on this stimulus, brain waves in visual cortex could be observed with matching frequencies. To date, BCI still faces challenges and a major limitation is the EEG poor spatial resolution. This limitation is due to the volume conductance effect [15], as the skull bones act as a non-linear low pass filter, mixing the brain source signals and thus reducing the signal-to-noise ratio.

Consequently, spatial filtering methods are used, such as xDAWN [17], Independent Component Analysis (ICA) [20], Common Spatial Pattern (CSP) [10] and Canonical Correlation Analysis (CCA) [12]. Spatial filters, obtained by diagonalization of data covariance matrices, enhance the differences between variances of signals of different classes/tasks. They are efficient on clean datasets obtained from strongly constrained environment. However they are sensitive to artifacts and outliers $[13,19]$. Working directly on covariance matrices is advantageous: it simplifies the whole BCI system [21], avoiding the alignment of two learning steps (spatial filters and classifiers) that might lead to overfitting. Covariance matrices being Symmetric and Positive-Definite (SPD), they are best handled by tools provided by Riemannian geometry. Classification in the space of SPD matrices eliminates the need of spatial filters and improves the system robustness $[5,7,21]$.

A classification technique referred to as minimum distance to Riemannian mean (MDRM) has been recently introduced to EEG classification [5]. It entirely relies on covariance matrices and the fact that they belong to the manifold of SPD matrices. New EEG trials are assigned to the class whose average covariance matrix is the closest to the trial covariance matrix according to the affine-invariant Riemannian metric [14]. It is a simple, yet robust classification scheme outperforming complex and highly parametrized state-of-the-art classifiers. The limitations of using Euclidean metrics in the computation of distances between SPD matrices and their means have been demonstrated [3]. Using information geometry, a number of Riemannian distances have been developed and appropriately used on SPD matrices $[1,3]$. The present work applies some of these distances to SSVEP data, providing a practical analysis and a comparison with Euclidean distance.

Moreover, most applications of Riemannian geometry to BCI are thus far focusing only on Motor Imagery (MI) paradigm. Riemannian BCI is well suited for MI experiment as the spatial information linked with synchronization are directly embedded in covariance matrices obtained from multichannel recordings. However, for BCI that rely on evoked potential such as SSVEP or event-related potential, as P300, frequency or temporal information are needed. In [7], the authors propose a rearrangement of the covariance matrices that embed the timing or the frequency information, thus allowing a direct application of the Riemannian framework. This contribution relies on this rearrangement to apply MDRM on covariance matrices of SSVEP signals. The signals are recorded in an application of assistive robotics where an SSVEP-based BCI is used in tandem 
with a 3D touchless interface based on IR-sensors as a multimodal system to control an arm exoskeleton [11].

The paper is organized as follows: Section 2 describes the framework for the classification of covariance matrices. The distances and means considered for this study are presented. In Section 3, the classification results obtained on real EEG dataset are presented and discussed. Section 4 concludes this paper.

\section{Classification of covariance matrices for SSVEP}

A SSVEP classifier based on covariance matrices is presented. The computation of means of training covariance matrices is crucial to the classifier performance.

\subsection{Means for covariance matrices}

In the following, we will consider covariance matrices belonging to the manifold $\mathcal{M}_{C}$ of the $C \times C$ symmetric positive definite matrices, defined as:

$$
\mathcal{M}_{C}=\left\{\Sigma \in \mathbb{R}^{C \times C}: \Sigma=\Sigma^{\top} \text { and } x^{\top} \Sigma x>0, \forall x \in \mathbb{R}^{C} \backslash 0\right\} .
$$

Given a set of covariance matrices $\left\{\Sigma_{i}\right\}_{i=1, \ldots, I}$, we consider the mean matrix $\bar{\Sigma}$ of the set, which is a covariance matrix that minimizes the sum of the squared distances to matrices $\Sigma_{i}$ :

$$
\bar{\Sigma}=\mu\left(\Sigma_{1}, \ldots, \Sigma_{I}\right)=\arg \min _{\Sigma \in \mathcal{M}_{C}} \sum_{i=1}^{I} d^{m}\left(\Sigma_{i}, \Sigma\right),
$$

where $m=1$ when $d(\cdot, \cdot)$ is a divergence (i.e. a generalization of squared distance), and $m=2$ when $d(\cdot, \cdot)$ is a distance.

From Eq. (1), several means can be defined and those considered in this study are indicated in Table 1 . We consider the Euclidean distance $d_{\mathrm{E}}$, as a baseline, yielding the arithmetic mean. The first considered Riemannian distance is the Log-Euclidean $d_{\mathrm{LE}}$ distance. Its mean is expressed explicitly [3]. The second is the Affine-Invariant $d_{\mathrm{AI}}$ [14]. Unlike the $d_{\mathrm{LE}}$, it does not have an explicit expression for the mean. It could be efficiently computed with the gradient-based iterative algorithm proposed in [8]. The two last distances considered in this study are the log-determinant $\alpha$-divergence [6] and the Bhattacharyya distance [16], the later being a special case of the the former with $\alpha=0$. Since the $\alpha$-divergence is not symmetrical, its right version is used in this work [6].

\subsection{Minimum Distance to Mean classifier for SSVEP}

The considered classifier is referred to as Minimum Distance to Mean (MDM), and is inspired from [5] where it is limited to Riemannian mean. Covariance

\footnotetext{
${ }^{4}$ For $\alpha=-1$, the log-determinant $\alpha$-divergence is defined as: $\operatorname{tr}\left(\Sigma_{1}^{-1} \Sigma_{2}-I\right)-$ $\log \operatorname{det}\left(\Sigma_{1}^{-1} \Sigma_{2}\right)$, and for $\alpha=1: \operatorname{tr}\left(\Sigma_{2}^{-1} \Sigma_{1}-I\right)-\log \operatorname{det}\left(\Sigma_{2}^{-1} \Sigma_{1}\right)[6]$.
} 


\begin{tabular}{|l|c|c|c|}
\cline { 2 - 4 } \multicolumn{1}{c|}{} & Distance/Divergence & Mean & References \\
\hline Euclidean & $d_{\mathrm{E}}\left(\Sigma_{1}, \Sigma_{2}\right)=\left\|\Sigma_{1}-\Sigma_{2}\right\|_{F}$ & $\bar{\Sigma}_{\mathrm{E}}=\frac{1}{I} \sum_{i=1}^{I} \Sigma_{i}$ & \\
Log-Euclidean & $d_{\mathrm{LE}}\left(\Sigma_{1}, \Sigma_{2}\right)=\left\|\log \left(\Sigma_{1}\right)-\log \left(\Sigma_{2}\right)\right\|_{F}$ & $\bar{\Sigma}_{\mathrm{LE}}=\exp \left(\sum_{i=1}^{I} \log \left(\Sigma_{i}\right)\right)$ & {$[2,3]$} \\
Affine-invariant & $d_{\mathrm{AI}}\left(\Sigma_{1}, \Sigma_{2}\right)=\left\|\log \left(\Sigma_{1}^{-1} \Sigma_{2}\right)\right\|_{F}$ & Algorithm 3 in $[8]$ & {$[14,8]$} \\
$\alpha$-divergence $\left({ }^{4}\right)$ & $\begin{array}{c}d_{\alpha \mathrm{D}}\left(\Sigma_{1}, \Sigma_{2}\right)=\frac{4}{1-\alpha^{2}} \log \frac{\operatorname{det}\left(\frac{1-\alpha}{2} \Sigma_{1}+\frac{1+\alpha}{2} \Sigma_{2}\right)}{\operatorname{det}\left(\Sigma_{1}\right)^{\frac{1-\alpha}{2}} \operatorname{det}\left(\Sigma_{2}\right)^{\frac{1+\alpha}{2}}} \\
\text { Bhattacharyya }\end{array}$ & Algorithm 1 in $[6]$ & {$[6]$} \\
\hline $\mathrm{B}\left(\Sigma_{1}, \Sigma_{2}\right)=\left(\log \frac{\operatorname{det} \frac{1}{2}\left(\Sigma_{1}+\Sigma_{2}\right)}{\left(\operatorname{det}\left(\Sigma_{1}\right) \operatorname{det}\left(\Sigma_{2}\right)\right)^{1 / 2}}\right)^{1 / 2}$ & Algorithm 1 in $[6]$ & {$[6,16]$} \\
\hline
\end{tabular}

Table 1. Distances, divergences and means considered in the experimental study.

matrices of EEG trials are classified based on their distance to the centers of the classes, equal to means. To embed frequency information in the covariance matrices, we use a construction of matrices proposed in [7]. Let $X \in \mathbb{R}^{C \times N}$ be an EEG trial measured on $C$ channels and $N$ samples in a SSVEP experiment with $F$ stimulus blinking at different frequencies. The covariance matrices are estimated from a modified version of the input signal $X$ :

$$
X \in \mathbb{R}^{C \times N} \rightarrow\left[\begin{array}{c}
X_{\mathrm{freq}_{1}} \\
\vdots \\
X_{\mathrm{freq}_{F}}
\end{array}\right] \in \mathbb{R}^{F C \times N}
$$

where $X_{\text {freq }_{f}}$ is the input signal $X$ band-pass filtered around frequency freq , $_{f}$ $f=1, \ldots, F$. Henceforth, all EEG signals will be considered as filtered and modified by Eq. (2). The associated covariance matrix $\Sigma \in \mathcal{M}_{F C}$ is estimated using the Schäfer shrinkage estimator [18].

For SSVEP classification, $K=F+1$ classes are considered: one class for each target frequency, and one for the resting state. As described in Algorithm 1, from $I$ labelled training trials $\left\{X_{i}\right\}_{i=1}^{I}$ recorded per subject, $K$ centers of classes $\bar{\Sigma}^{(k)}$ are estimated (step 3 ). In this step, outlier matrices are removed to have a reliable mean estimation, using an offline Riemannian potato [4]. A new unlabeled test trial $Y$ is predicted to belong to the class whose mean $\bar{\Sigma}^{(k)}$ is the closest to the trial covariance matrix, w.r.t. one of the distances from Table 1 (step 5). Remark that test trial has to be finished before being classified: in this paper, there is no early classification.

\section{Experimental Results}

This section presents experimental results obtained applying Euclidean and Riemannian distances in SSVEP classification task. The first part of this section describes the data used and the second part provides the assessment of the classification for the considered distances and divergences. 


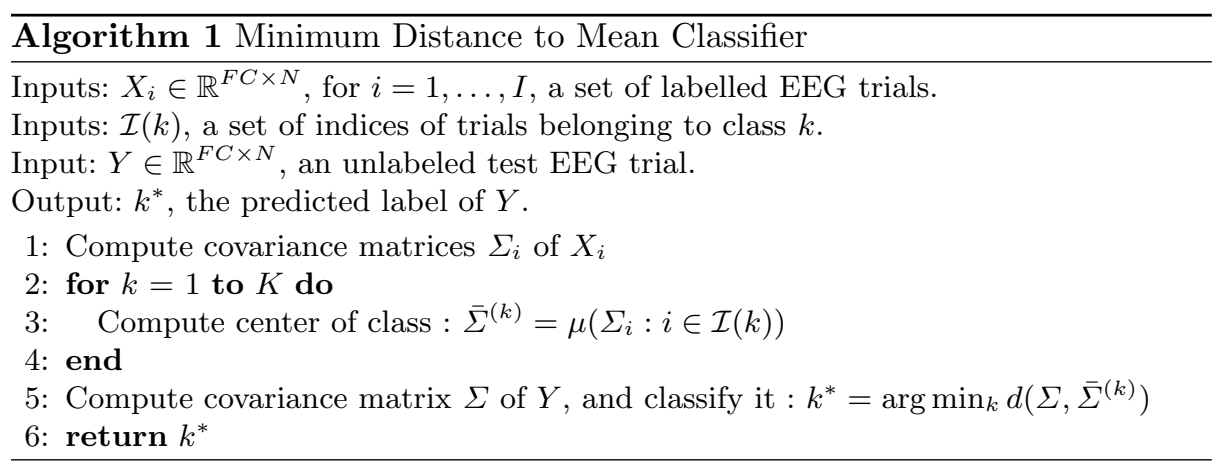

\subsection{SSVEP Dataset}

The experimental study is conducted on multichannel EEG signals recorded during an SSVEP-based BCI experiment [11]. EEG are recorded on $C=8$ channels (i.e. Oz, O1, O2, PO3, POz, PO7, PO8, PO4) from 12 subjects. The subjects are presented with $F=3$ visual target stimuli blinking respectively at $13 \mathrm{~Hz}, 17 \mathrm{~Hz}$ and $21 \mathrm{~Hz}$. It is a $K=4$ classes setup combining $F=3$ stimulus classes and one resting class (no-SSVEP). In a session, 32 trials are recorded: 8 for each visual stimulus and 8 for the resting class. The number of sessions recorded per subject varies from 2 to 5 . For each subject, a test set is made of 32 trials while the remaining trials (which might vary from 32 to 128) make up for the training set.

\subsection{Results and Discussion}

The MDM classifier is simple. Once the covariance matrices have been estimated, the only major calculations involved are the mean and distance computations. The covariance matrices obtained from SSVEP data extended with Eq. (2) have interesting features, allowing the discrimination between signals of identical sources but with different frequencies. Fig. 1 shows the $K$ classes mean covariance matrices $\bar{\Sigma}^{(k)}$ from subjects with the highest (a) and lowest (b) classification accuracies. The three $8 \times 8$ diagonal blocks hold the covariance matrices of the $F=3$ target frequencies. Inter-frequencies covariances blocks are almost null. In each mean covariance matrix, the block holding the covariance of the target frequency has the largest values. For the resting class, all $F$ blocks tend to have similar and small values. These features are more visible in the subject with the highest classification accuracy, and less visible in the one with lowest classification accuracy. Contrary to discriminative classifiers classically used in BCI, such as LDA or SVM [9] which can appear as black-boxes with difficult interpretation, it is very interesting to see that the presented covariance based classifier uses features with a simple representation, and thus allows for an intuitive understanding. The observed covariance matrices have a physiological meaning and interpretation. In this framework, EEG processing complexity is encoded by a dedicated distance and not by a machine learning algorithm. 

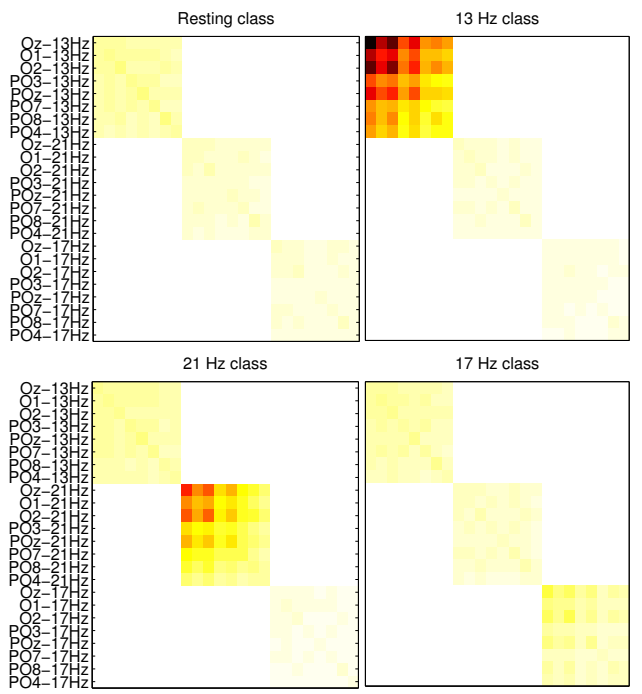

(a)

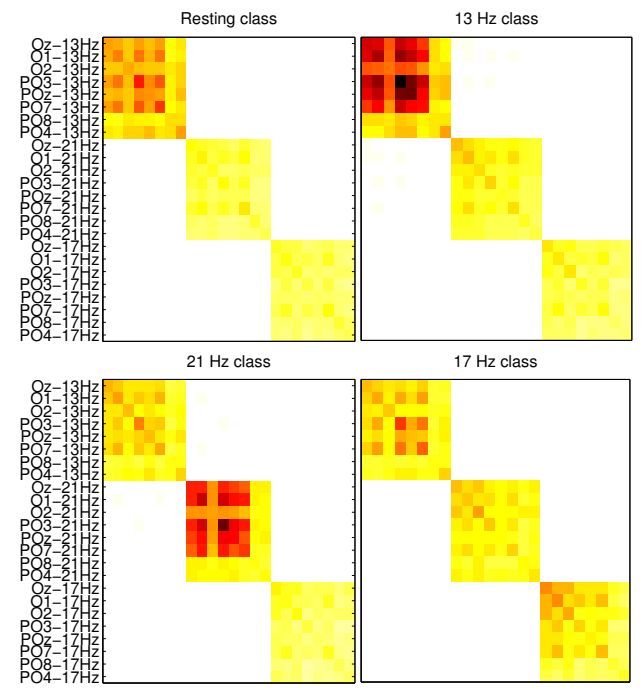

(b)

Fig. 1. Representation of covariance matrices: each image is the covariance matrix mean $\bar{\Sigma}^{(k)}$ of the class $k$, for one session of the recording. The diagonal blocks show the covariance in different frequency bands, i.e. $13 \mathrm{~Hz}$ in the upper-left block, $21 \mathrm{~Hz}$ in the middle, and $17 \mathrm{~Hz}$ in the bottom-right. The two chosen subjects are those with the highest (a) and the lowest (b) BCI performance. 


\begin{tabular}{|c|c|c|c|c|c|c|c|c|c|c|c|}
\hline & \multirow{2}{*}{\begin{tabular}{|c|}
$\mathrm{CCA}+\mathrm{SVM}$ \\
{$[12]$} \\
\end{tabular}} & \multirow{2}{*}{\multicolumn{2}{|c|}{$\begin{array}{l}\text { Euclidean } \\
\text { arithmetic }\end{array}$}} & \multicolumn{8}{|c|}{ Riemannian } \\
\hline & & & & Log-Ev & lidean & Affine-Ir & nvariant & $\alpha$-diver & rgence & Bhattac & haryya \\
\hline Sub. & $\operatorname{acc}(\%)$ & $\operatorname{acc}(\%)$ & time $(\mathrm{s})$ & $\operatorname{acc}(\%)$ & time $(\mathrm{s})$ & $\operatorname{acc}(\%)$ & time(s) & $\operatorname{acc}(\%)$ & $\operatorname{time}(\mathrm{s})$ & $\operatorname{acc}(\%)$ & time(s) \\
\hline 1 & 54.68 & 53.12 & 0.025 & 71.88 & 0.150 & 73.44 & 0.194 & 59.37 & 0.155 & 68.75 & 0.225 \\
\hline 2 & 37.50 & 43.75 & 0.020 & 78.13 & 0.160 & 79.69 & 0.190 & 79.69 & 0.200 & 81.25 & 0.065 \\
\hline 3 & 89.06 & 67.19 & 0.020 & 85.94 & 0.120 & 85.93 & 0.205 & 95.31 & 0.155 & 85.94 & 0.100 \\
\hline 4 & 79.69 & 54.68 & 0.030 & 84.38 & 0.225 & 87.50 & 0.315 & 89.07 & 0.250 & 85.94 & \begin{tabular}{|l|l|}
0.100 \\
\end{tabular} \\
\hline 5 & 50.00 & 37.50 & 0.020 & 62.50 & 0.115 & 68.75 & 0.290 & 73.44 & 0.140 & 65.62 & 0.125 \\
\hline 6 & 87.50 & 34.37 & 0.015 & 84.38 & 0.120 & 85.94 & 0.210 & 87.50 & 0.145 & 82.81 & 0.100 \\
\hline 7 & 77.08 & 60.42 & 0.027 & 87.50 & 0.267 & 88.54 & 0.410 & \begin{tabular}{|l|}
91.66 \\
\end{tabular} & 0.417 & 86.46 & 0.137 \\
\hline 8 & 73.44 & 67.19 & 0.035 & 90.63 & 0.215 & 92.19 & 0.290 & 92.19 & 0.290 & 92.19 & 0.125 \\
\hline 9 & 60.94 & 57.81 & 0.035 & 70.31 & 0.275 & 70.31 & 0.380 & 75.00 & 0.300 & 67.19 & \begin{tabular}{|l|l|l}
0.134 \\
\end{tabular} \\
\hline 10 & 67.97 & 38.28 & 0.035 & 75.00 & 0.254 & 80.47 & 0.514 & 82.03 & 0.510 & 78.13 & 0.160 \\
\hline 11 & 71.88 & 48.44 & 0.025 & 60.94 & 0.144 & 65.63 & 0.235 & 57.81 & 0.150 & 75.00 & 0.105 \\
\hline 12 & 95.63 & 71.25 & 0.032 & 96.25 & 0.292 & 96.69 & 0.534 & 95.62 & 0.634 & 96.88 & 0.300 \\
\hline Avg. & 70.45 & 52.83 & 0.027 & 78.98 & 0.194 & 81.27 & 0.314 & 81.56 & 0.279 & 80.51 & 0.140 \\
\hline
\end{tabular}

Table 2. Subject classification accuracies $(\operatorname{acc}(\%))$ and average CPU time (time(s)) elapsed for the classification of a single trial. Classification is performed with MDM using either Euclidean or Riemannian means (see Table 1). Results obtained with a state-of-the-art method using CCA and SVM [12] are included.

Based on those covariance matrices, the different distances and means of Table 1 are compared in terms of classification accuracy and average CPU time elapsed on a trial classification, which involves the computation of 4 means of class and a distance to each mean. One can note that for an optimal implementation, the 4 means are computed only once. Table 2 summarizes results obtained for each subject and each distance/divergence. Results obtained with a state-of-the-art method are also included, combining CCA and SVM [12].

Euclidean distance yields drastically low accuracy. This supports the fact that using Euclidean distance and arithmetic mean on SPD matrices is not appropriate. This is generally attributed to the invariance under inversion that is not guaranteed (i.e. $\left.\bar{\Sigma}\left(\Sigma_{i}\right) \neq \bar{\Sigma}^{-1}\left(\Sigma_{i}^{-1}\right)\right)$ and the fact that the determinant of the arithmetic mean of SPD matrices can be larger than the determinant of its parts; it is referred to as the swelling effect. Since the value of the determinant is a direct measure of dispersion of the multivariate variables (i.e. EEG channels and frequency bands), it leads to poor discrimination in the classification task. The swelling effect of arithmetic mean is shown in Fig. 3.2: the determinant of the arithmetic mean is strictly larger than other means, the Log-Euclidean, Affine-Invariant and Bhattacharyya ones yielding similar determinants, close to trials values.

Using Riemannian distances significantly improves classification performances, with regards to state-of-the-art method (70.45\%) and Euclidean distance. The $\alpha$-divergence yields the best results $(81.56 \%)$. The value of $\alpha$ was set to 0.6 through cross-validation. This procedure lasted 225.42 seconds and makes $\alpha$ divergence the most costly method, due to the optimization of its parameter $\alpha$. 


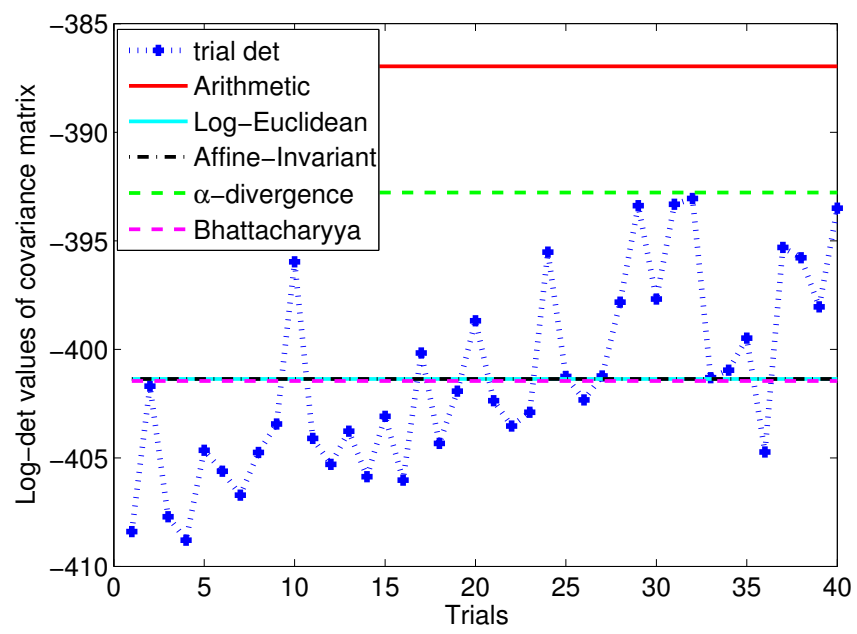

Fig. 2. (a): Swelling effect of arithmetic mean shown through log-determinant values. Training trials are taken from the $13 \mathrm{~Hz}$ class of the subject with the highest BCI performance. Log-determinant values are given for each trial covariance (points), and for means of Table 1 (horizontal lines).

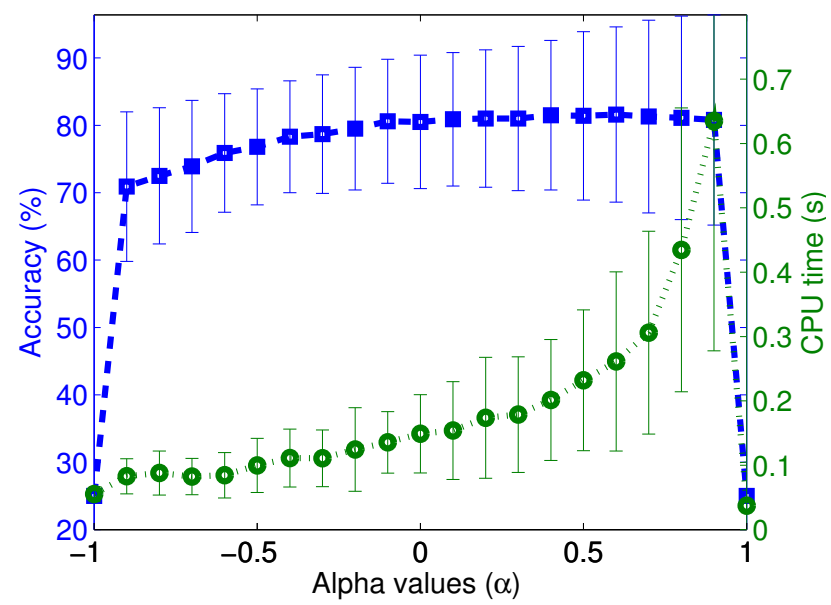

Fig. 3. Classification accuracy and CPU time obtained for the log-determinant $\alpha$ divergence, with $-1 \leqslant \alpha \leqslant 1$. The values are averaged across all sessions and subjects.

Log-Euclidean yields lower classification accuracy (average 78.98\%) but could be computed faster than $\alpha$-divergence or Affine-Invariant distance. However, the Bhattacharyya distance has the lowest computational cost of the considered Riemannian distances (average CPU time 0.140s), with a higher average accuracy of $80.51 \%$. So, it is good trade-off between efficiency and speed. The 
accuracies and CPU time of the $\alpha$-divergence at different values of $\alpha$ are shown in Fig. 3.2. It is seen that for $\alpha= \pm 1$, where $\alpha$-divergence represents a Bregman divergence associated with the log-determinant function, the classification accuracy are drastically low $(25 \%)$. For the rest, the accuracy varies smoothly with changes in $\alpha$, with the highest accuracy scored while $\alpha$ is positive.

This experiment on real EEG data shows that it is crucial to process covariance matrices with dedicated Riemannian tools, impacting the efficiency of the classification.

\section{Conclusion}

Riemannian approaches have been successfully applied on EEG signals for brain computer interfaces. Straightforward algorithms, such as Minimum Distance to Mean, provide competitive results with state-of-the-art methods, without requiring meticulous parametrization or optimization. Working on covariance matrices in Riemannian spaces offers a wide choice of distances, embedding desirable invariances: it is thus possible to avoid the computation of user-specific spatial filters which are sensitive to artifacts and outliers. Nonetheless, the estimation of the Riemannian geometric mean has a strong impact on the classifier accuracy. This study investigates the performance of several distances and divergence on a real EEG dataset in the context of BCI based on the SSVEP paradigm. The experimental results indicate that the $\alpha$-divergence yields the best accuracy after the selection of the best $\alpha$ values, but the Bhattacharyya distance has the lowest computational cost while providing decent accuracies.

\section{References}

1. Amari, S.I.: $\alpha$-divergence is unique, belonging to both $f$-divergence and Bregman divergence classes. IEEE Trans Inf Theory 55(11), 4925-4931 (2009)

2. Arsigny, V., Fillard, P., Pennec, X., Ayache, N.: Log-Euclidean metrics for fast and simple calculus on diffusion tensors. Magn. Reson. Med. 56(2), 411-421 (2006)

3. Arsigny, V., Fillard, P., Pennec, X., Ayache, N.: Geometric means in a novel vector space structure on symmetric positive-definite matrices. SIAM Journal on Matrix Analysis and Applications 29(1), 328-347 (2007)

4. Barachant, A., Andreev, A., Congedo, M., et al.: The Riemannian potato: an automatic and adaptive artifact detection method for online experiments using Riemannian geometry. In: Proceedings of TOBI Workshop IV. pp. 19-20 (2013)

5. Barachant, A., Bonnet, S., Congedo, M., Jutten, C.: Multiclass brain-computer interface classification by Riemannian geometry. IEEE Trans Biomed Eng 59(4), 920-928 (2012)

6. Chebbi, Z., Moakher, M.: Means of Hermitian positive-definite matrices based on the log-determinant $\alpha$-divergence function. Linear Algebra and its Applications 436(7), 1872-1889 (2012)

7. Congedo, M., Barachant, A., Andreev, A.: A new generation of brain-computer interface based on Riemannian geometry. arXiv preprint arXiv:1310.8115 (2013) 
8. Fletcher, Joshi, S.: Principal geodesic analysis on symmetric spaces: Statistics of diffusion tensors. In: Computer Vision and Mathematical Methods in Medical and Biomedical Image Analysis, LNCS, vol. 3117, pp. 87-98. Springer (2004)

9. Hastie, T., Tibshirani, R., Friedman, J., Hastie, T., Friedman, J., Tibshirani, R.: The elements of statistical learning, vol. 2. Springer (2009)

10. Johannes, M.G., Pfurtscheller, G., Flyvbjerg, H.: Designing optimal spatial filters for single-trial EEG classification in a movement task. Clinical Neurophysiology 110(5), 787-798 (1999)

11. Kalunga, E.K., Chevallier, S., Rabreau, O., Monacelli, E.: Hybrid interface: Integrating BCI in multimodal human-machine interfaces. In: Advanced Intelligent Mechatronics (AIM), 2014 IEEE/ASME Int. Conf. on. pp. 530-535. IEEE (2014)

12. Kalunga, E.K., Djouani, K., Hamam, Y., Chevallier, S., Monacelli, E.: SSVEP enhancement based on Canonical Correlation Analysis to improve BCI performances. In: AFRICON, 2013. pp. 1-5. IEEE (2013)

13. Lotte, F., Guan, C.: Regularizing Common Spatial Patterns to Improve BCI Designs: Unified Theory and New Algorithms. Biomedical Engineering, IEEE Transactions on 58(2), 355-362 (2011)

14. Moakher, M.: A differential geometric approach to the geometric mean of symmetric positive-definite matrices. SIAM Journal on Matrix Analysis and Applications 26(3), 735-747 (2005)

15. Niedermeyer, E., da Silva, F.L.: Electroencephalography: Basic Principles, Clinical Applications, and Related Fields. Lippincott Williams \& Wilkins, 5th edn. (2004)

16. Nielsen, F., Bhatia, R.: Matrix Information Geometry. Springer Publishing Company, Incorporated (2012)

17. Rivet, B., Souloumiac, A., Attina, V., Gibert, G.: xDAWN algorithm to enhance evoked potentials: Application to brain-computer interface. IEEE Trans Biomed Eng 56(8), 2035-2043 (2009)

18. Schäfer, J., Strimmer, K.: A shrinkage approach to large-scale covariance matrix estimation and implications for functional genomics. Statistical applications in genetics and molecular biology 4(1) (2005)

19. Tomioka, R., Aihara, K., Müller, K.R.: Logistic regression for single trial EEG classification. In: NIPS. vol. 19, pp. 1377-1384 (2007)

20. Wang, S., James, C.J.: Enhancing evoked responses for BCI through advanced ICA techniques. In: MEDSIP. pp. 1-4 (2006)

21. Yger, F.: A review of kernels on covariance matrices for BCI applications. In: Machine Learning for Signal Processing (MLSP), 2013 IEEE International Workshop on. pp. 1-6. IEEE (2013) 